\title{
Will SARS-CoV-2 variants of concern affect the promise of vaccines?
}

\section{Ravindra K. Gupta}

Initial optimism regarding the development of COVID-19 vaccines has been tempered by the emergence of new variants of SARS-CoV-2. Will vaccination be able to contain the pandemic? How can we best optimize the limited supplies of vaccines available and what should future COVID-19 vaccines look like?

Amongst the fear and uncertainty during the early months of the coronavirus disease 2019 (COVID-19) pandemic, enormous hope was placed in vaccines, the development of which progressed at unprecedented speed throughout 2020 . The first clinical trials were started in March 2020 (of Moderna's mRNA-1273 vaccine) and the first licenses were granted in December 2020 (for mRNA vaccines from Moderna and Pfizer/ BioNTech and the viral-vectored AstraZeneca vaccine). These achievements were the result of decades of scientific research in infectious diseases and vaccinology, latterly involving the development of adenoviral vectors and mRNA technology. The former had already been used in candidate vaccines for the related betacoronavirus MERS-CoV and for Ebola. mRNA-based vaccines are a more recent innovation, having originally been developed for use in cancer, although not used for infections previously.

Early hopes that vaccination might provide a longterm solution to the pandemic were bolstered by the reported slow mutation rate of severe acute respiratory syndrome coronavirus 2 (SARS-CoV-2), which was estimated from global phylogenies to accumulate approximately two mutations per month. At such a rate, immune escape was not considered to be a serious threat. By mid-2020, however, there were anecdotal reports of some individuals with chronic shedding of virus, followed by case reports of viruses with multiple mutations emerging in individuals with chronic infection in the context of an immunosuppressive state. Several of the mutations in the SARS-CoV-2 spike protein that were observed in these cases, for example at position E484 in the receptor-binding domain (RBD) of the spike protein, had already been predicted to affect the binding of monoclonal antibodies and also to escape neutralization by convalescent sera. How do these emerging viral variants of concern impact on the hopes of long-term pandemic control through vaccination?

The B.1.1.7 variant (also known as 501Y.V1, having a N501Y mutation in the RBD) emerged in the UK in the second half of 2020 , with the first sequences detected in September 2020 (REF. ${ }^{1}$ ). This variant has eight spike mutations that include two deletions, one of which is in an antibody supersite epitope (Y144) and the other of which increases infectivity but has little impact on immune escape ${ }^{1}$. The sole RBD mutation is N501Y, which also seems to increase binding to the host cell receptor ACE2. Epidemiological analyses quickly ascertained that B.1.1.7 was more transmissible ${ }^{2}$. So far, the B.1.1.7 variant has spread globally and has been noted to also acquire the E484K mutation in the UK and USA. There was estimated to be a 6 -fold decrease in sensitivity of this E484K-mutant virus to immune sera from individuals vaccinated with the Pfizer/BioNTech mRNA vaccine and an 11-fold decrease in sensitivity to convalescent sera ${ }^{3}$.

The B.1.351 and P.1 variants (also known as 501Y. V2 and 501Y.V3) that have emerged in South Africa and Brazil, respectively, have caused the greatest anxiety, owing to the co-occurrence of additional mutations in the RBD at positions E484 and K417. Viral variants with the triple combination of N501Y, E484K and K417N/T have significantly reduced susceptibility to vaccine-induced and convalescent sera ${ }^{4}$. Worryingly, there is evidence for convergent evolution of mutations such as N501Y and E484K, for example not only in the variants discussed above but also in other variants of concern such as P.3 in the Philippines, B.1.526 in the USA and B.1.525 in the UK and west Africa.

In addition to these RBD mutations, a neglected area of SARS-CoV-2 evolution pertains to the importance of amino-terminal domain (NTD) mutations in spike protein, given the 242 base-pair deletion in B.1.351, as well as H69/V70 and Y144 deletions in B.1.1.7. The NTD mutation L18F is also increasing in frequency in B.1.1.7 and is found in B.1.351 viruses. These NTD mutations confer reduced sensitivity to neutralizing antibodies. In addition, although deletion at H69/V70 does not seem to confer antibody evasion, it makes SARS-CoV-2 more permissive for deleterious escape mutations in the RBD, such as the mink-related mutation $\mathrm{Y}_{453 \mathrm{~F}^{1}}$. The mechanism seems to involve greater incorporation of spike 
protein into virions. This brings us to the important question of whether vaccination could be used to protect against such viral variants that are able to increase their infection advantage? For example, the D614G mutation, located in the S2 domain of spike protein, was acquired by SARS-CoV-2 in early 2020 and this variant spread globally within months. D614G is now present in nearly all new infections, including all variants of concern. Deletion at H69/V70 is reminiscent of the D614G mutation in terms of its effect on increasing viral fitness, so perhaps as a next step in vaccine design we should be seeking ways to block these fitness-enhancing mutations.

In vitro neutralization assays have suggested that many of these variants of concern have reduced sensitivity to vaccine-induced and convalescent sera, but what do we know so far about how they affect vaccine efficacy in the field? Which vaccines have been tested in populations where the three main variants of concern (B.1.1.7, B.1.351 and P.1) are transmitting? Of considerable reassurance are the emerging data showing protection from severe infection and death for all vaccines in all settings, although the prevention of asymptomatic transmission and mild-to-moderate disease is more variable. The AstraZeneca ChAdOx1 vaccine showed only $10 \%$ protection against mild-to-moderate disease associated with the B.1.351 variant in a young population with median age of 30 in South Africa ${ }^{5}$. By contrast, in the UK, ChAdOx1 demonstrated $75 \%$ protection against B.1.1.7 (including asymptomatic infection). The Novavax vaccine, which consists of purified spike protein, showed approximately $50 \%$ protection against infection in South Africa (largely the B.1.351 variant) and $86 \%$ protection against infection in the UK (predominantly the B.1.1.7 variant) ${ }^{6}$. Johnson \& Johnson's human adenovirus-vectored vaccine showed $64 \%$ protection against moderate-to-severe disease in South Africa (dominated by the B.1.351 variant) and $66 \%$ protection against moderate-to-severe disease in the USA (mainly the Wuhan-1 variant with D614G), as assessed 29 days after vaccination ${ }^{7}$. The Pfizer/BioNTech BNT162b2 mRNA vaccine was reported to be less effective against B.1.351 than against non-B.1.351 variants based on a small analysis of breakthrough infections that were enriched for B.1.351 in Israel ${ }^{8}$. The efficacy of CoronaVac/Sinovac inactivated virus vaccine in Brazil, where $75 \%$ of infections were with the P.1 variant, was estimated at around 50\% against symptomatic infection ${ }^{9}$.

In summary, we still need more information regarding the protection from infection and disease that is afforded by the current generation of SARS-CoV-2 vaccines in the light of the existing and potentially newly emerging viral variants of concern. Further work on the role of T cell-mediated immunity to SARS-CoV-2 and its role in selecting for viral variants is also needed. Another key question is whether we need to develop and implement modified vaccines sooner rather than later? Indeed, the National Institutes of Health in the USA recently funded a safety and immunogenicity study of Moderna's mRNA1271.351 vaccine, which has been designed against the B.1.351 variant that emerged in South Africa, including E484K, K417N and N501Y mutations. However, the mutations that would need to be included in a modified vaccine are debatable. I would suggest, for example, E484K, N501Y and L452R mutations in the RBD (L452R being found in the recently reported B.1.617 variant emerging in India and in the B.1.429 variant that has emerged in the USA), and I would also consider P681H/R mutation in the furin cleavage site (which is found in the B.1.1.7 variant and the B.1.617 variant) as well as NTD deletions. In my view, there should be a regularly updated consensus document from the World Health Organization that details the mutations to be included in the next generation of vaccines and guidelines on how to use them. I anticipate, based on SARS-CoV-2 evolution so far, that yearly revision of the vaccine sequence will be needed for the next 2-3 years at least to include emerging mutations. Continued emphasis on limiting transmission through non-pharmaceutical interventions will also be crucial to reduce the opportunity for novel variants to emerge, including 'super variants' that might arise from chronic infection with an existing variant of concern.

Given the slow progress on global vaccine coverage so far, there are calls to extend dosing schedules from 4 weeks to 12 weeks between first and second doses, as has been implemented in the UK, to maximize the number of protected individuals and break chains of transmission. However, data from single-dose mRNA vaccination suggest that in the elderly (more than 80 years of age), this would lead to significant proportions of individuals having non-protective levels of neutralizing antibodies for an additional 8 weeks ${ }^{10}$. T cell responses may protect against severe disease in these individuals, but onward viral transmission from poor neutralizers is possible during this extended window. Finally, we will also need to think about how we might protect against SARS-CoV-2 variants in those individuals who are immunosuppressed and therefore unable to mount responses even after two vaccine doses.

1. Kemp, S. A. et al. Recurrent emergence and transmission of a SARS-CoV-2 spike deletion H69/V70. Preprint at bioRxiv https:// doi.org/10.1101/2020.12.14.422555 (2021).

2. Volz, E. et al. Assessing transmissibility of SARS-CoV-2 lineage B. 1.1.7 in England. Nature https://doi.org/10.1038/s41586-021 03470-x (2021).

3. Collier, D. A. et al. Sensitivity of SARS-CoV-2 B.1.1.7 to mRNA vaccine-elicited antibodies. Nature https://doi.org/10.1038/ s41586-021-03412-7 (2021).

4. Cele, S. et al. Escape of SARS-CoV-2 501Y.V2 from neutralization by convalescent plasma. Nature https://doi.org/10.1038/s41586-021. 03471-w (2021).

5. Madhi, S. A. et al. Efficacy of the ChAdOx 1 nCoV-19 Covid-19 vaccine against the B. 1.351 variant. N. Engl. J. Med. https://doi.org/ 10.1056/NEJMoa2102214 (2021).

6. Shinde, V. et al. Preliminary efficacy of the NVX-CoV2373 Covid-19 vaccine against the B.1.351 variant. Preprint at medRxiv https:// doi.org/10.1101/2021.02.25.21252477 (2021).

7. Sadoff, J. et al. Safety and efficacy of single-dose Ad26.COV2.S vaccine against Covid-19. N. Engl. J. Med. https://doi.org/10.1056/ NEJMoa2101544 (2021).

8. Kustin, T. et al. Evidence for increased breakthrough rates of SARS-CoV-2 variants of concern in BNT162b2 mRNA vaccinated individuals. Preprint at medRxiv https://doi.org/10.1101/ 2021.04.06.21254882 (2021).

9. Hitchings, M. D. T. et al. Effectiveness of CoronaVac in the setting of high SARS-CoV-2 P. 1 variant transmission in Brazil: a test-negative case-control study. Preprint at medRxiv https://doi.org/10.1101/ 2021.04.07.21255081 (2021).

10. Collier, D. A. et al. Age-related heterogeneity in immune responses to SARS-CoV-2 vaccine BNT162b2. Preprint at medRxiv https:// doi.org/10.1101/2021.02.03.21251054 (2021).

Competing interests

R.K.G. has received consultancy fees from Janssen related to work on vaccines. 